\title{
PATTERN DETECTION USING A MODIFIED COMPOSITE FILTER WITH NONLINEAR JOINT TRANSFORM CORRELATOR.
}

\author{
S. Vallmitjana, I. Juvells, A. Carnicer and J. Campos. \\ Universitat de Barcelona. \\ Laboratori d'Optica. Departament de Física Aplicada i Electrònica. \\ Diagonal 647. 08028 Barcelona. SPAIN.
}

\begin{abstract}
A simple method for optical implementation of a composite filter in a joint transform correlator is presented. A composite filter in object space is a real function, but by adding an appropriate constant value to it, only positive values are obtained. As a drawback, the bias introduced decreases the relative height of the correlation peaks in the recognition process.

Non-linear effects in a joint transform correlator are used to increase discrimination by using positive composite filters. Digital simulation and optical implementation of the process has been carried out, achieving the non-linearities by means of saturation effects on the intensity detection process.
\end{abstract}

\section{INTRODUCTION}

The matched filter technique, introduced by Vander Lugt ${ }^{1}$, was conceived to recognize a specific image. A large signal in presence of the image to be detected is produced and also a weak one in the presence of another image. The matched filter has great limitations because it has a low discrimination capability between similar images, and is not invariant for rotation, distortion or contrast changes in the image to be detected.

The composite filter, also called synthetic discriminant function (SDF), introduced by Caulfield and Maloney in $1969^{2,3}$ and subsequently developed by Casasent and co-workers ${ }^{4,5}$, was conceived to increase the performance of the matched filter. Using this type of filter it is possible to enhance the between-class discrimination capabilities and decrease the within-class 
classification error. It is also possible to achieve different types of recognition performances: rotation, distortion or contrast invariance ${ }^{6}$.

The composite filter is essentially obtained by linear combination of different filters associated with several images. The corresponding coefficients are calculated by imposing the values of the correlation between the SDF and each image is considered. In general, if the initial images and the correlation values imposed are real, the coefficient of the linear combination and the SDF, in object space, will also be real, normally taking both positive and negative values.

For optical implementation of a SDF in a joint transform correlator ${ }^{7}$ (JTC), one possibility is to codify positive and negative values of the filter by techniques of digital holography ${ }^{8}$. Another possibility is to add a constant value to the filter function, so that only positive values are obtained. The filter will then be performed by a photographic register of a display of this non-negative function. This is a very simple method but it suffers from the disadvantage that the bias introduced decreases the relative height of the correlation peaks in the recognition process.

Non-linear effects in a JTC can be used to increase discrimination in a recognition process. ${ }^{9}$. In this study we present a simple method for optical implementation of an SDF in a JTC. This modified filter (PSDF, positive synthetic discriminant function) is obtained by adding a positive constant value to the classical composite filter, corresponding to its minimum value. A digital simulation of the method has been carried out and the results obtained have been corroborated by the implementation of the PSDF in an experimental set-up. The filter is registered photographically and used as reference in a JTC that includes a CCD camera that projects the power spectrum to a low-cost liquid crystal television (LCTV). Non-linearities have been introduced by saturating the CCD-LCTV ensemble in order to increase the performance of the JTC.

\section{COMPOSITE FILTER DESIGN}

The composite filter technique can be used to improve the performances of a conventional matched filter. In this work we use two types of composite filters, one is designed to recognize an object while rejecting all others. A second composite filter is designed to recognize two objects together and to discriminate the others. 
A composite filter $f(x, y)$ is a linear combination of targets of the form

$$
f(x, y)=a_{1} g_{1}(x, y)+\ldots .+a_{n} g_{n}(x, y)
$$

where $g_{i}(x, y)$ are two-dimensional functions representing the differents objects considered and $a_{i}$ are the relative weights of the linear combination. These coefficients are obtained by imposing the values of the correlation at the origin between the filter $f(x, y)$ and each target $g_{i}(x, y)$.

$$
\begin{array}{lll}
c_{i}=\left[g_{i}(x, y) \otimes f(x, y)\right](0,0) & =1 & \text { if filter detects } g_{i}(x, y) \\
& =0 & \text { if filter rejects } g_{i}(x, y)
\end{array}
$$

where $\mathrm{i}=1, \ldots, \mathrm{n}$

By using expression (1) in filter conditions, we obtain the following equations:

$$
\begin{gathered}
a_{1} c_{11}(0,0)+\ldots+a_{n} c_{1 n}(0,0)=c_{1} \\
\ldots \ldots \\
a_{n} c_{n 1}(0,0)+\ldots+a_{n} c_{n n}(0,0)=c_{n}
\end{gathered}
$$

where $c_{i j}$ are the cross-correlations (at the origin) between the target $g_{i}(x, y)$ and $g_{j}(x, y)$.

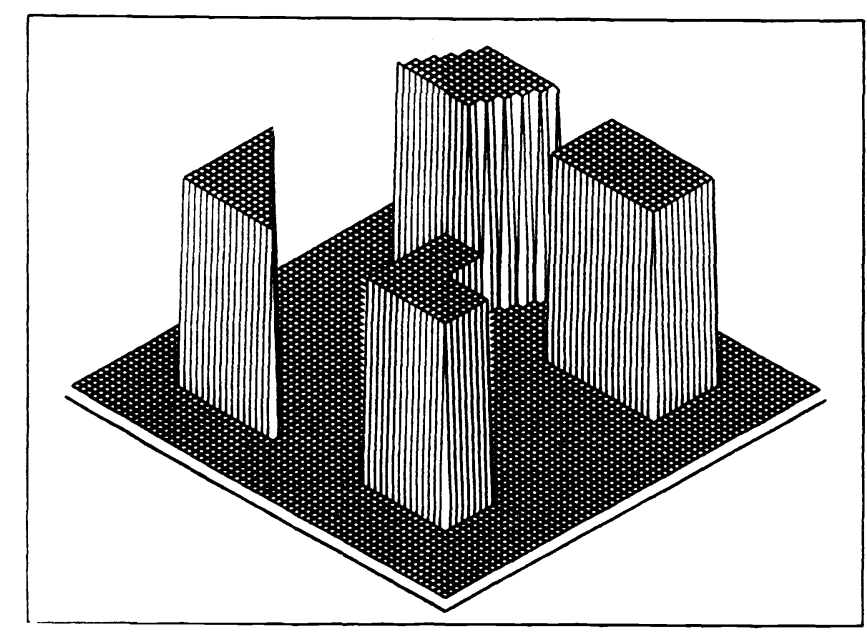

Figure 1: Scene.

Finally, solving the equations of system (3), $a_{i}$ coefficients are obtained.

The scene used is shown in figure 1. The first positive composite filter is designed to recognize the triangle, rejecting the rectangle, the rhombus and the angle form and the second filter is designed to recognize the triangle and the rhombus together, rejecting the rectangle and the angle. 


\section{COMPUTER SIMULATION}

In this paragraph we show the analysis of the non-linearity effects in a SDF by digital simulation. Two composite filters with the desired performance that are lineal combinations of the four objects present in the scene (fig. 1), have been designed:

a) Filter $f_{1}(x, y)$ : classical SDF adapted to recognize the triangle. Its correlation at the origin with the triangle is made equal to 1 , and the response to the other equal to 0 , as in equation (2).

b) Filter $f_{2}(x, y)$ : classical SDF adapted to recognize the triangle and the rhombus. Its correlations at the origin with the triangle and rhombus are made equal to 1 and the correlations with the other objects are equal to 0 . These two objects have been chosen because their cross-correlation are the lowest and so represent the most difficult case.

These filters present positive and negative values in different zones. Figures $2 a$ and $2 b$ show the correlations between the scene and filters $f_{1}$ and $f_{2}$ respectively. With the composite filter method it is not possible to prevent large sidelobe levels from occurring in the correlation plane ${ }^{10}$, as it can be seen in fig. 2a. This behaviour is stronger with filter $f_{2}$.

To implement a SDF in an amplitude optical modulator or photographic register, filter amplitude transmission should be non-negative. The easiest way is to add a constant bias value to the filter, obtaining a positive synthetic discrimination function (PSDF). Figures $3 a$ and $3 b$ show the bias addition effect on the correlation. Contrast decreases and detection and discrimination capability diminishes. If this kind of non-negative filters is used in an optical correlator the detection process will be almost impossible.

It has been shown previously ${ }^{9}$ that a binary joint transform correlator (BJTC) may produce reasonably good correlation performance in terms of correlation peak intensity, peak-to-sidelobe ratio and correlation width. An important parameter to choose in BJTC is the threshold level to binarize the joint power spectrum. In the literature this level is usually selected by making an histogram of the pixel values of the interference intensity and then picking the median. In order to increase discrimination capability and prevent false alarms ${ }^{11}$, we have found that selecting a level of $30 \%$ of pixels in 1 is a better choice ${ }^{12}$. 

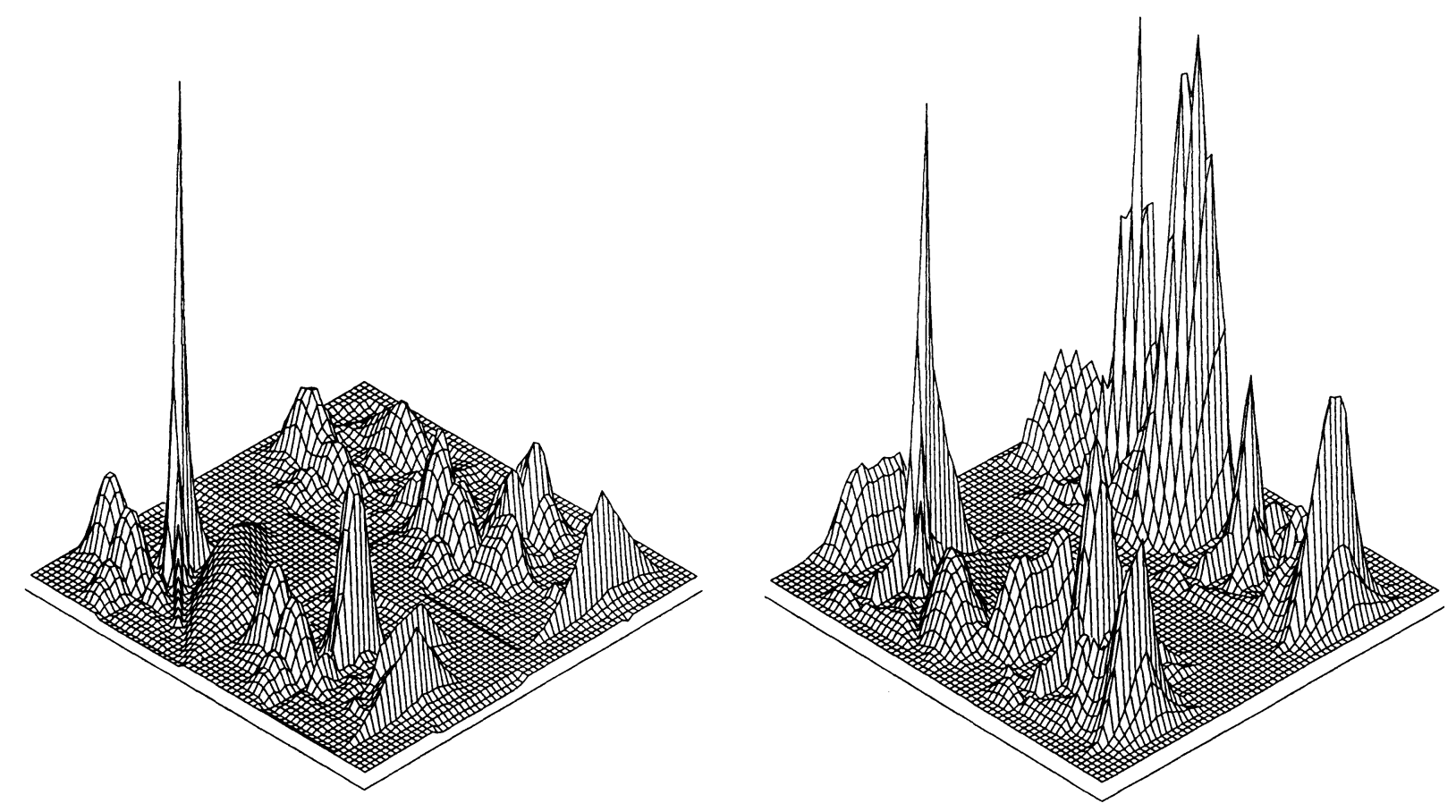

Figure 2: Cross-correlation with SDF:

a) designed to detect triangle

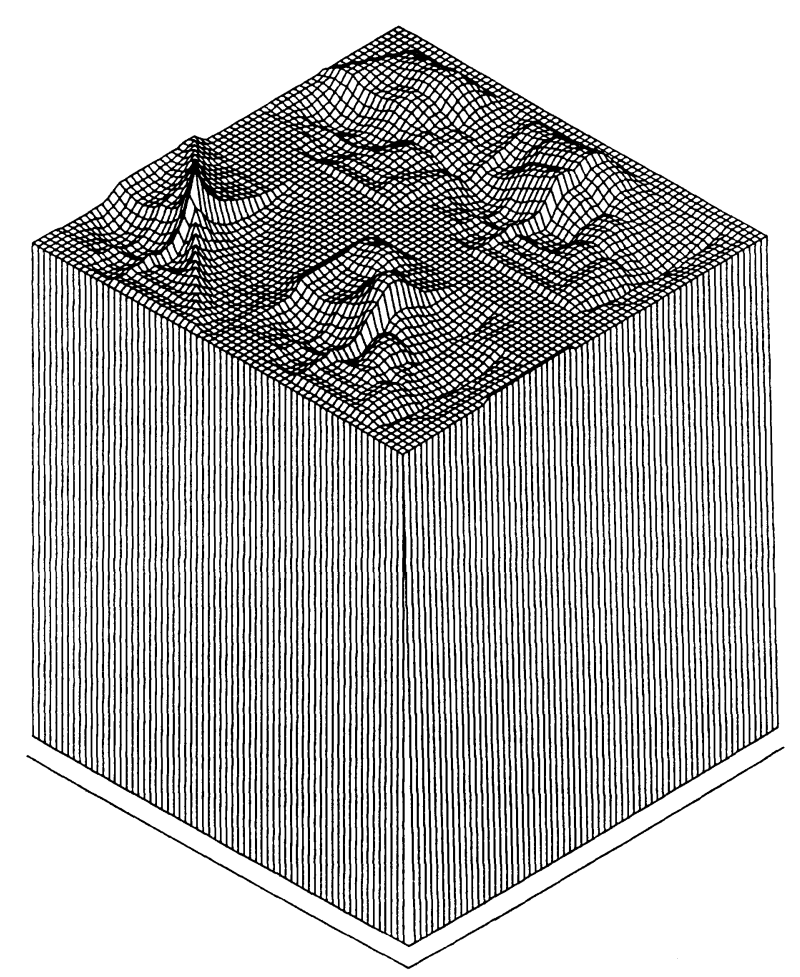

b) designed to detect triangle and rhombus.

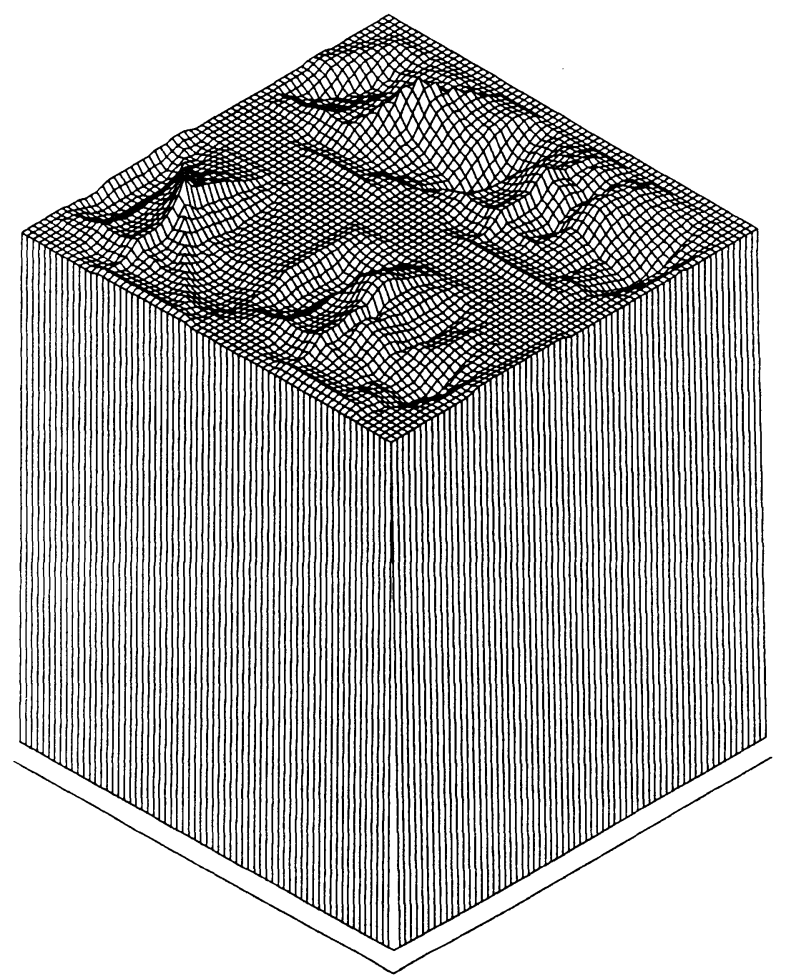

Figure 3: Cross-correlation with PSDF:

a) designed to detect triangle

b) designed to detect triangle and rhombus. 

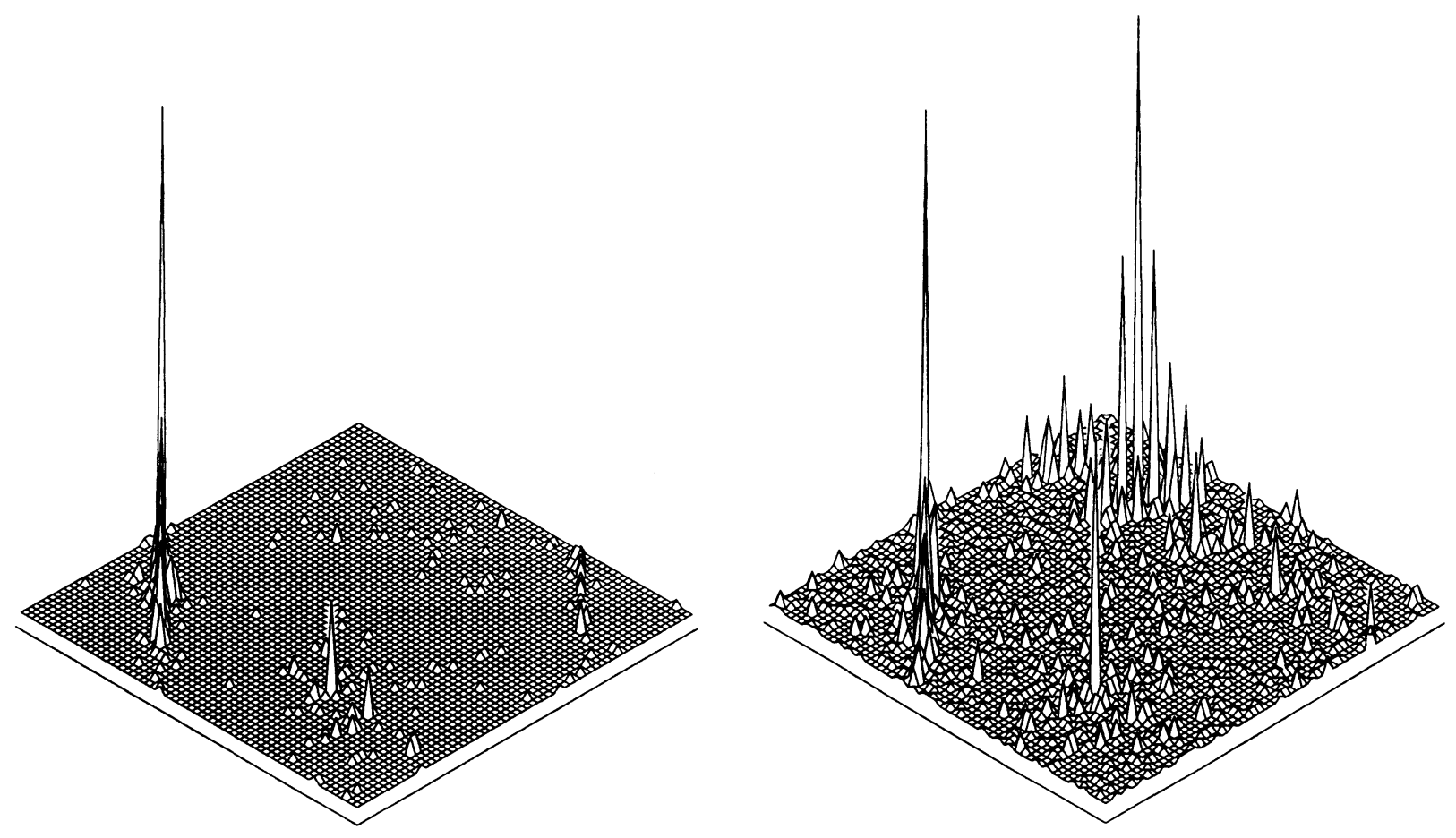

Figure 4: Cross-correlation with PSDF and binarized power spectrum:

a) designed to detect triangle

b) designed to detect triangle and rhombus.
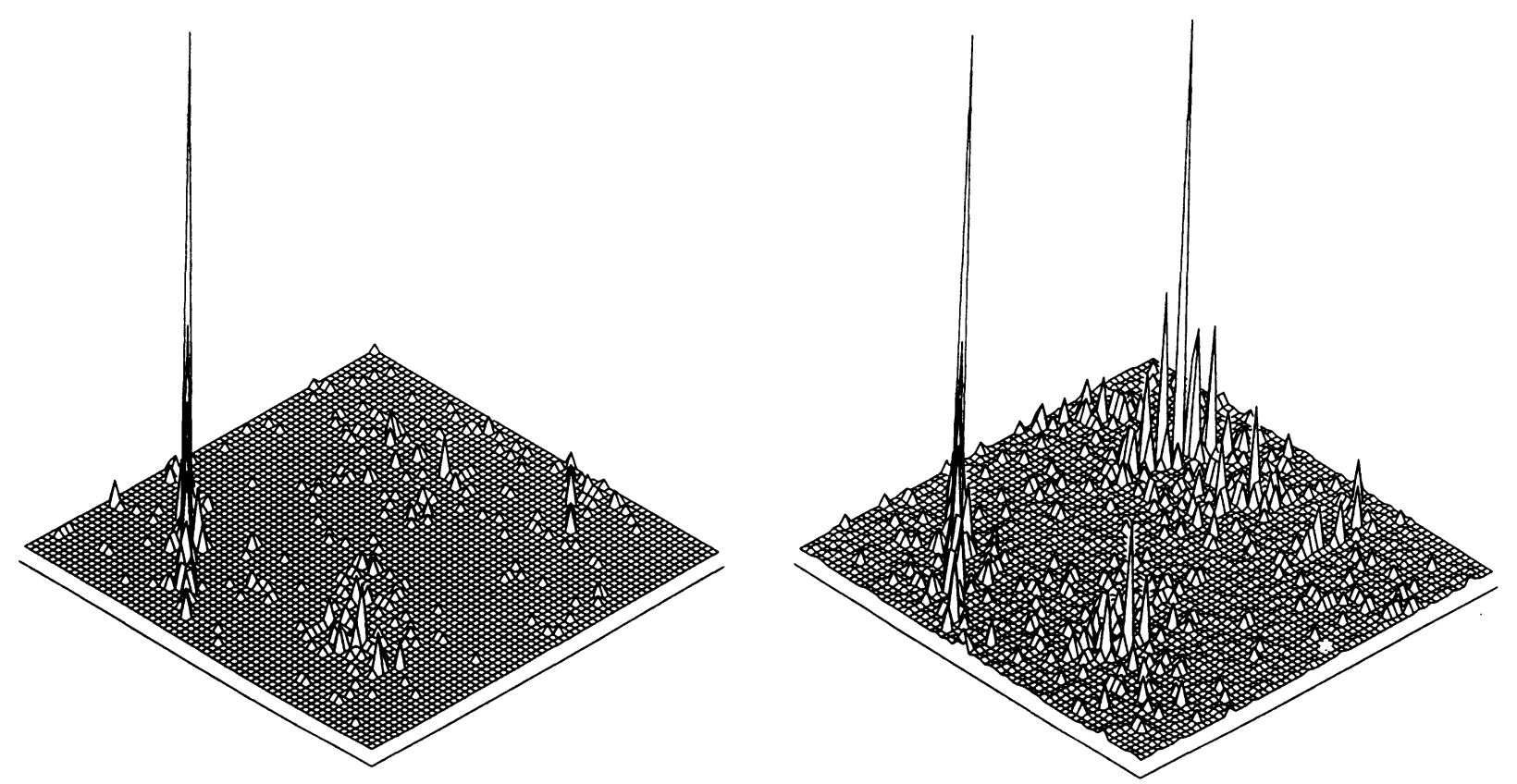

Figure 5: Cross-correlation with OPSDF and binarized power spectrum: a) designed to detect triangle

b) designed to detect triangle and rhombus. 
Figures $4 \mathrm{a}$ and $4 \mathrm{~b}$ show the correlation results obtained with a BJTC by using the composite filters $f_{1}$ and $f_{2}$, with the added constant being positive (PSDF). Comparing these results with those obtained with SDF (fig. 2) it is possible to see, that discrimination capability increases and sidelobes decrease, showing the good performance of the method. Nevertheless, by using filter $f_{2}$, the peak corresponding to the angle is not negligible because this object is very similar to the triangle.

As we have mentioned above, the bias introduced to obtain PSDF causes a decrease in discrimination ability as shown in fig. 3. In an attempt to reduce the bias effect and to prevent high sidelobes values, we use an optimized version of the PSDF (OPSDF). In PSDF, we add a constant value to all the filters. Now, this value is only added to the signal $f(x, y)$. The computer simulation results obtained by using an OPSDF in a BJTC are shown in figures 5a and 5b. A comparison of this figure with figure 4 shows that the sidelobes and the height of the correlation peak corresponding to the angle in figure $5 \mathrm{~b}$, are lower.

\section{EXPERIMENTAL}

A joint transform correlator was assembled with a CCD camera for the detection of the intensity of the Fourier plane and an adapted commercial liquid crystal television (B/W LCTV Casio-200) introduces a transmittance proportional to this intensity into a second optical Fourier transformer. The correlation is obtained in its output plane. Scene and filter were introduced into the input plane by means of a photographic record, in well-controlled conditions in order to obtain a transmission proportional to the calculated values.

We have recently verified that if light forming the first Fourier transform is augmented, so that the couple CCD-LCTV is saturated, the discrimination in the output plane is increased. In this way saturation is an easy method to introduce the non-linear effects of binarization simulated in the previous section into the optical set-up. The number of grey levels in the transmission of the LCTV in the second transformer decreases with the saturation in a such way that it can be considered binary.

Figure $6 \mathrm{a}$ and $6 \mathrm{~b}$ show the experimental correlation results obtained by using OPSDF $\mathrm{f}_{1}$ and $f_{2}$ and saturing the power spectrum. Saturation was performed in a way that a ratio of black and white transmission in the LCTV was approximately the same as in the simulation process. 

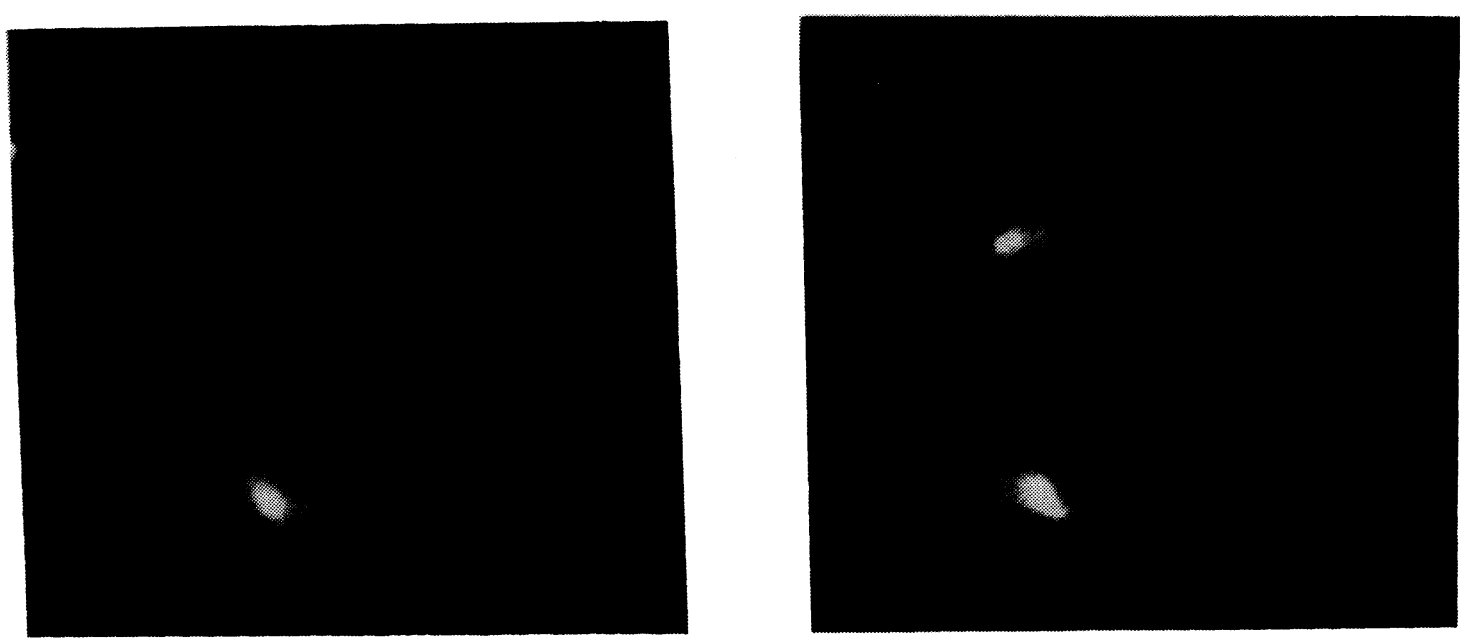

Figure 6: Experimental cross-correlation with OPSDF and saturated power spectrum:

a) designed to detect triangle

b) designed to detect triangle and rhombus.

\section{SUMMARY}

We have proposed a simple method to obtain a positive synthetic discriminant function (SDF), for optical implementation, based on the addition of a constant. Although the discrimination in the direct cross-correlation is worse, it is shown that the binarization in the power spectrum improves the discrimination with the composite filter. We also have evidenced an optimization of this technique by adding only the constant value to the signal filter. The results have been computer simulated and carried out experimentally in a JTC

\section{REFERENCES}

1. A.B. Vander Lugt, "Signal detection by complex spatial filtering", IEEE Trans. Inf. Theory IT-10, 139-145 (1964).

2. H.J. Caulfield and W.T. Maloney, "Improved discrimination in optical character recognition", Appl. Opt., 8, 2354-2356 (1969).

3. H.J. Caulfield, "Linear combinations of filters for character recognition: a unified treatment", Appl. Opt., 19, 3877-3879 (1980).

4. C.F. Hester and D. Casasent, "Multivariant technique for multiclass pattern recognition", Appl. Opt., 19, 1758-1761 (1980). 
5. D. Casasent, "Unified synthetic discriminant function computational formulation", App. Opt., 23, 1620-1627 (1984).

6. H.H. Arsenault, L. Leclerc and Y. Sheng, "Similarity and invariance in pattern recognition", Proc. SPIE, 960, 2-17 (1988).

7. F.T.S. Yu and H.J. Lu, "A real-time programmable joint transform correlator", Opt. Commun., 52, 10-16 (1984).

8. S. Vallmitjana, J. Campos, I. Juvells and S. Bosch, "Optical implementation of a composite filter using a joint-transform correlator", Proc. SPIE, 1134, 119-125 (1989).

9. B. Javidi, "Nonlinear joint power spectrum based optical correlations", Appl. Opt., 28, 2358-2367 (1989).

10. D. Casasent and W. T. Chang, "Correlation synthetic discriminant functions", Appl. Opt., 25, 2343-2350 (1986).

11. F. T. S. Yu, F. Cheng, T. Nagata and D. A. Gregory, "Effects of fringe binarization of multiobject joint transform correlation", Appl. Opt., 28, 2988-2990 (1989).

12. A. Carnicer, I. Juvells and S. Vallmitjana, "Effects of thresholding level variation in fringe binarization of multiobject joint transform correlation", submitted to Appl. Opt. 